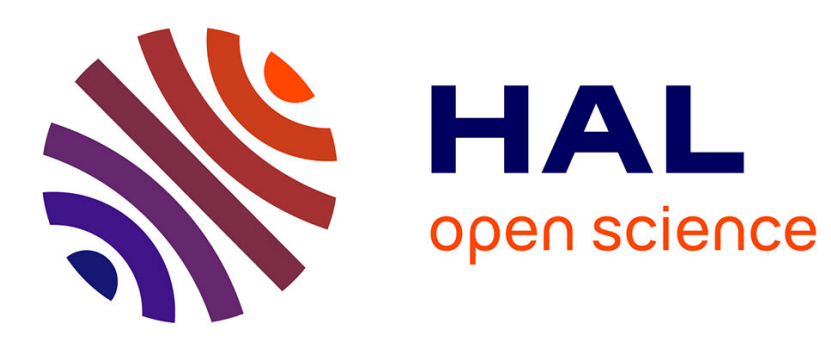

\title{
Optimal control separating two microalgae species competing in a chemostat
} Walid Djema, Olivier Bernard, Térence Bayen

\section{To cite this version:}

Walid Djema, Olivier Bernard, Térence Bayen. Optimal control separating two microalgae species competing in a chemostat. 2020, 2020 59th IEEE Conference on Decision and Control (CDC), 10.1109/CDC42340.2020.9304115 . hal-02939495

\section{HAL Id: hal-02939495 \\ https://hal-univ-avignon.archives-ouvertes.fr/hal-02939495}

Submitted on 15 Sep 2020

HAL is a multi-disciplinary open access archive for the deposit and dissemination of scientific research documents, whether they are published or not. The documents may come from teaching and research institutions in France or abroad, or from public or private research centers.
L'archive ouverte pluridisciplinaire HAL, est destinée au dépôt et à la diffusion de documents scientifiques de niveau recherche, publiés ou non, émanant des établissements d'enseignement et de recherche français ou étrangers, des laboratoires publics ou privés. 


\title{
Optimal control separating two microalgae species competing in a chemostat
}

\author{
Walid Djema* $\quad$ Olivier Bernard ${ }^{* * *} \quad$ Térence Bayen***
}

\begin{abstract}
In this work, we study the question of selecting in minimal-time a microalgae species of interest using the Droop model to describe the dynamics of two distinct populations competing for a limiting nutrient. This amounts to consider an optimal control problem governed by a five-dimensional affine control system in which the control is the dilution rate. Throughout this paper, the optimal control strategies allowing the strain of interest to dominate the population in minimaltime is discussed. These results are illustrated using a numerical direct optimization method (implemented in Bocop).
\end{abstract}

Index Terms-Optimal control, Pontryagin Maximum Principle, Singular arc, Droop's model, Chemostat system.

\section{INTRODUCTION}

Microbial ecology relies on the concept of ecosystem, which is defined as a complex association of microorganisms in a specific environment and their non-microbial surroundings [1]. There is a strong mutual relationship between microorganisms and their environment. In particular, the influence of the habitat on microorganism-residents can be manipulated and exploited to guide their future. This concept applies well to microalgae, which are unicellular photosynthetic microorganisms of major importance, due to their applications in various fields [2]. Microalgae can be used for producing compounds of interests like proteins, pigments, antioxidants or essential fatty acids ([3]). At longer term, their use for $\mathrm{CO}_{2}$ mitigation and bioenergy production has been also considered, even if one faces many challenges to decrease cost and environmental impact [4]. The biotechnological use of microalgae is still very young, and in practice, only a few wild species are used among the large microalgal biodiversity. Compared to agriculture where domesticated species resulted from centuries of selection and hybridization, there is still a long way to go in order to select more productive microalgae. Selecting and improving these microorganisms is however challenging due to their complex interaction with their environmental conditions. Selection by Darwinian pressure, while imposing conditions that favor the species of interests has given spectacular results (see, e.g., [5]). These continuous-selection approaches are however time consuming, with experiments that can last several months to several years. We can expect to improve these

\footnotetext{
${ }^{*}$ Côte d'Azur University (UCA), Inria Sophia-Antipolis Méditerranée, Biocore project-team, France.

** Laboratoire d'Océanographie de Villefranche (LOV), Université de Sorbonne, CNRS, Observatoire Océanologique, Villefranche-sur-Mer, France.

***Avignon Université, Laboratoire de Mathématiques d'Avignon (EA 2151), F-84018 Avignon, France.

Corresponding author: walid.djema@inria.fr

olivier.bernardeinria.fr

terence.bayen@univ-avignon.fr
}

experiments by optimal control, especially playing on the dilution rate of the photobioreactor. One main issue is then to identify strategies minimizing the operating time when the species of interest dominates the population. There are several works based of optimal control theory to study the selection of a species of interest in minimal-time. For the chemostat system under Monod's kinetics, this problem has been addressed in [6], [7] by studying a two-dimensional system. It appears that microalgae are better represented by Droop's model (leading to a five-dimensional controlled system) which accounts for the internal accumulation of the limiting nutrient ([9], [10]). The minimal-time problem with Droop's model has been tackled by [11], [12] in a simplified case in which the model dimension has been partially reduced. In this paper, we focus on the problem of selecting the species of interest in minimal-time with two Droop-species. Optimal strategies to let the species of interest dominate are derived using the Pontryagin maximum principle (PMP, see [13]).

The paper is structured as follows : the optimal control problem (OCP) is stated in Section II] in which Droop's model, the control system, and the target are introduced. Pontryagin's principle is used in Section III in order to give properties of an optimal control of the studied $\mathrm{OCP}^{1}$. Singular arcs and Legendre-Clebsch's conditions are discussed in Section IV Finally, in Section V, we depict numerically optimal strategies using a direct method in optimal control.

\section{STATEMENT OF THE OPTIMAL CONTROL PROBLEM}

\section{A. Droop's model}

We consider the so-called Droop's model for two species, $x_{1}$ and $x_{2}$ competing on a limiting substrate $s$. Their growth depends on an intracellular quota of the limiting nutrient ([9]) denoted $q_{1}$ and $q_{2}$, respectively:

$$
\left\{\begin{aligned}
\dot{s} & =\left(s_{\text {in }}-s\right) D(t)-\rho_{1}(s) x_{1}-\rho_{2}(s) x_{2}, \\
\dot{q}_{1} & =\rho_{1}(s)-\mu_{1}\left(q_{1}\right) q_{1} \\
\dot{x}_{1} & =\left[\mu_{1}\left(q_{1}\right)-D(t)\right] x_{1} \\
\dot{q}_{2} & =\rho_{2}(s)-\mu_{2}\left(q_{2}\right) q_{2} \\
\dot{x}_{2} & =\left[\mu_{2}\left(q_{2}\right)-D(t)\right] x_{2}
\end{aligned}\right.
$$

where $s_{i n}$ is the constant input concentration of the substrate. The chemostat dilution rate $D(\cdot)$ is a bounded nonnegative control function such that $D(t) \in\left[0, D_{\max }\right]$, where $D_{\max }>$ 0 is the maximum capacity of the feeding pump, above

\footnotetext{
${ }^{1}$ Optimal Control Problem
} 
the maximum effective growth rate of the two species as illustrated in Figure 1 (see also [12]). In addition, for $i=1,2$, $\rho_{i}$ is a real-valued function quantifying the rate of substrate absorption, i.e. the uptake rate of the free nutrient $s$ ([14]), and $\mu_{i}$ is a real-valued function quantifying the growth rate of the $i$-th species. In the rest of the paper, we suppose that the uptake rates $\rho_{i}(s)$ are expressed in terms of MichaelisMenten's kinetics (see, e.g., [14]),

$$
\rho_{i}(s)=\frac{\rho_{m_{i}} s}{K_{s_{i}}+s},
$$

where $K_{s i}$ is a strictly positive constant standing for the $i$ th species. We also suppose that for each species, there is a minimum threshold $k_{q_{i}}>0$, under which cell division cannot occur. For Droop's model, the kinetics $\mu_{i}$ are given by,

$$
\mid \begin{array}{ll}
\mu_{i}\left(q_{i}\right)=0 & 0 \leq q_{i} \leq k_{q_{i}}, \\
\mu_{i}\left(q_{i}\right)=\mu_{i \infty}\left(1-\frac{k_{q_{i}}}{q_{i}}\right) & q_{i} \geq k_{q_{i}} .
\end{array}
$$

We now wish to state an invariance property for (1). Doing so, let us introduce the following notation. Set,

$$
M_{i}:=\sup _{s \in\left[0, s_{i n}\right]} \rho_{i}(s)<\rho_{m_{i}}, \quad i=1,2,
$$

and let us uniquely define $\bar{q}_{1}$ and $\bar{q}_{2}$ such that $\mu_{i}\left(\bar{q}_{i}\right) \bar{q}_{i}=M_{i}$, $i=1,2$. Thus, we get the following invariance property.

Proposition 1: For every $q_{m_{i}} \geq \bar{q}_{i}, i=1,2$, the set

$$
\mathfrak{S}:=\left(0, s_{i n}\right) \times\left[k_{q_{1}}, q_{m_{1}}\right] \times \mathbb{R}_{+}^{*} \times\left[k_{q_{2}}, q_{m_{2}}\right] \times \mathbb{R}_{+}^{*},
$$

is forward invariant by (1).

The quantity $\bar{q}_{i}$ is the so-called maximum internal storage quota. In the sequel, we consider as invariant set the set $\mathfrak{S}$ with $q_{m_{1}}=\bar{q}_{1}$ and $q_{m_{2}}=\bar{q}_{2}$. We also suppose that $\rho_{1}$ and $\rho_{2}$ satisfy the following assumption:

Assumption 1: One has $\rho_{2}^{\prime \prime} \rho_{1}^{\prime}-\rho_{1}^{\prime \prime} \rho_{2}^{\prime}>0$.

In view of (2), one has,

$$
\rho_{2}^{\prime \prime}(s) \rho_{1}^{\prime}(s)-\rho_{1}^{\prime \prime}(s) \rho_{2}^{\prime}(s)=\frac{2 K_{s_{1}} K_{s_{2}} \rho_{m_{1}} \rho_{m_{2}}\left(K_{s_{2}}-K_{s_{1}}\right)}{\left(K_{s_{1}}+s\right)^{3}\left(K_{s_{2}}+s\right)^{3}},
$$

and therefore, Assumption 1 implies $K_{s_{2}}-K_{s_{1}}>0$.

\section{B. Mathematical problem formulation}

Throughout the paper, we consider that the first species (with concentration $x_{1}$ ) is the one of interest, and the objective is to find a feeding strategy, that is a control function $D(\cdot)$, such that $x_{1}$ becomes predominant in the bioreactor. This can be formulated in terms of the ratio between the concentrations, namely, we seek a control strategy $D(\cdot)$ for which at the end of the process, one has $\frac{x_{1}}{x_{2}} \gg 1$.

The set of admissible controls $D(\cdot)$ is defined as,

$$
\mathscr{D}:=\left\{D:[0,+\infty) \rightarrow\left[0, D_{\max }\right] ; D(\cdot) \in L_{l o c}^{\infty}\left(\mathbb{R}_{+}\right)\right\},
$$

In order to formalize the selection process between the two species, we consider a target set $\mathscr{T} \subset \mathfrak{S}$ as,

$$
\mathscr{T}=\left\{X:=\left(s, q_{1}, x_{1}, q_{2}, x_{2}\right) \in \mathfrak{S} ; x_{2} \leq \varepsilon x_{1}\right\} .
$$

Here, $0<\varepsilon \ll 1$ is a small parameter quantifying the contamination rate of the interesting strain $x_{1}$. Reaching $\mathscr{T}$ means that the biomass of the first species is significantly larger than the second one after a certain culture time. The optimal control problem can be then stated as follows. The objective is to determine a dilution-based control strategy $D \in \mathscr{D}$ that allows trajectories of (1) starting from any initial condition in $\mathfrak{S}$ to reach the target $\mathscr{T}$ in minimal-time, i.e.

$$
\inf _{D \in \mathscr{D}} t_{f}^{D} \quad \text { s.t. } X\left(t_{f}^{D}\right) \in \mathscr{T} \text { and } X^{0} \in \mathfrak{S},
$$

(OCP)

where $X(\cdot)$ is the unique solution of (1) associated with $D \in \mathscr{D}$ such that $X(0)=X^{0} \in \mathfrak{S}$. To ease the notation, we shall use in the following $t_{f}$ instead of $t_{f}^{D}$ (when there is no ambiguity on the control).

\section{Effective growth rates}

In this section, we introduce the effective growth rates that play a key role in the selection process. Let us start by introducing some notations. Firstly, observe that the choice of $\bar{q}_{i}, i=1,2$ implies that,

$$
0 \leq q_{i} \mu_{i}\left(q_{i}\right) \leq \bar{q}_{i} \mu_{i}\left(\bar{q}_{i}\right)<\rho_{m_{i}}
$$

for every $q_{i} \geq 0$, thus, the mapping,

$$
q_{i} \mapsto \delta_{i}\left(q_{i}\right):=\rho_{i}^{-1}\left(q_{i} \mu_{i}\left(q_{i}\right)\right)=\frac{K_{s_{i}} q_{i} \mu_{i}\left(q_{i}\right)}{\rho_{m_{i}}-q_{i} \mu_{i}\left(q_{i}\right)}
$$

is well defined over $\left[0, \rho_{m_{i}}\right)$ with values in $\left[0, s_{i n}\right)$, for $i=1,2$. Then, it follows that the mapping,

$$
s \mapsto \delta_{i}^{-1}(s):=\frac{k_{q_{i}} K_{s_{i}} \mu_{i \infty}+\left(\rho_{m_{i}}+\mu_{i \infty} k_{q_{i}}\right) s}{\mu_{i \infty}\left(K_{s_{i}}+s\right)},
$$

is well-defined over $\left[0, s_{i n}\right)$ with values in $\left[0, \rho_{m_{i}}\right)$.

Property 1: For $i=1,2$, one can readily check that the mappings $\delta_{i}$ and $\delta_{i}^{-1}$ are increasing over their definition sets.

It follows that for every $s \in\left[0, s_{i n}\right]$, one has $\delta_{i}(s)>k_{q_{i}}$, $i=1,2$. It is straightforward to check that for $i=1,2$ the effective growth rate is given by,

$$
\mu_{i}\left(\delta_{i}^{-1}(s)\right)=\frac{\rho_{m_{i}} \mu_{i \infty} s}{k_{q_{i}} K_{s_{i}} \mu_{i \infty}+\left(\rho_{m_{i}}+\mu_{i \infty} k_{q_{i}}\right) s} .
$$

Now, we introduce the function,

$$
\Delta(s):=\mu_{1}\left(\delta_{1}^{-1}(s)\right)-\mu_{2}\left(\delta_{2}^{-1}(s)\right), \quad s \in\left[0, s_{i n}\right],
$$

and we suppose that $\Delta$ satisfies the following characteristic:

Assumption 2: There exists a unique $\hat{s} \in\left(0, s_{\text {in }}\right)$ such that $\Delta(s)>0$ over $(0, \hat{s})$ and $\Delta(s)<0$ over $\left(\hat{s}, s_{i n}\right)$. In addition, $\Delta$ has a unique maximum $s_{c} \in[0, \hat{s}]$.

We also suppose that the maximum dilution rate is large enough to drive competition between species. This amounts to suppose that $D_{\max }$ satisfies the following assumption.

Assumption 3: The parameter $D_{\max }$ satisfies,

$$
D_{\max }>\max \left(\mu_{1}\left(\delta_{1}^{-1}(s)\right), \mu_{2}\left(\delta_{2}^{-1}(s)\right)\right), \quad s \in\left[0, s_{i n}\right] .
$$

These assumptions ensure reachability of the target and well-posedness of the studied optimization problem, since both species may win the competition (considering for instance a constant control parameter $D$ ). The resulting generic 

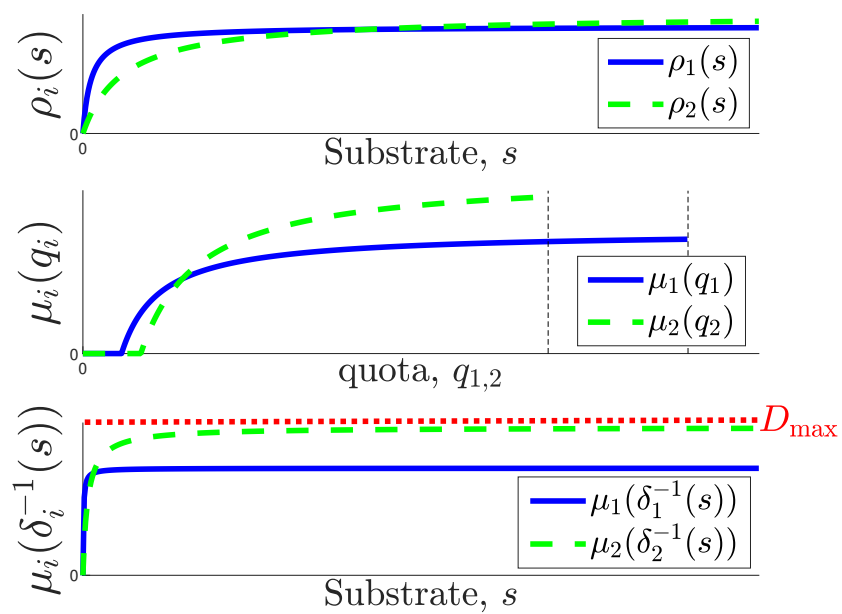

Fig. 1. The functions $\rho_{i}$ and $\mu_{i}$ satisfy Assumption 2. The choice of $D_{\max }$ above the maximum of the effective growth rates $\mu_{i}\left(\delta_{i}^{-1}(s)\right)$, for $i=1,2$, verifies the statement in Assumption 3.

functions are illustrated in Fig. 1] Considering an adequate constant control then allows indeed the system to select the first species of interest (by adapting the competitive exclusion principle [20]), but, this process is costly in time. Therefore, our objective in this paper is to introduce a dynamic strategy to reach the target set $\mathscr{T}$ faster, playing on the control $D(\cdot)$.

\section{NECESSARY OPTIMALITY CONDITIONS}

In this section, we apply the Pontryagin Maximum Principle (PMP) to derive necessary optimality conditions on optimal controls of (OCP). We denote by $X=\left(s, q_{1}, x_{1}, q_{2}, x_{2}\right)$ and $\lambda=\left(\lambda_{s}, \lambda_{q_{1}}, \lambda_{x_{1}}, \lambda_{q_{2}}, \lambda_{x_{2}}\right)$ respectively the state and adjoint variables. The Hamiltonian,

$$
H=H\left(s, q_{1}, x_{1}, q_{2}, x_{2}, \lambda_{s}, \lambda_{q_{1}}, \lambda_{x_{1}}, \lambda_{q_{2}}, \lambda_{x_{2}}, \lambda^{0}, D\right)
$$

associated with $\mathrm{OCP}$, is defined as,

$$
\begin{aligned}
& H=-\left(\rho_{1}(s) x_{1}+\rho_{2}(s) x_{2}\right) \lambda_{s}+\left(\rho_{1}(s)-\mu_{1}\left(q_{1}\right) q_{1}\right) \lambda_{q_{1}} \\
& +\mu_{1}\left(q_{1}\right) x_{1} \lambda_{x_{1}}+\left(\rho_{2}(s)-\mu_{2}\left(q_{2}\right) q_{2}\right) \lambda_{q_{2}}+\mu_{2}\left(q_{2}\right) x_{2} \lambda_{x_{2}} \\
& +\lambda^{0}+D\left[\left(s_{i n}-s\right) \lambda_{s}-x_{1} \lambda_{x_{1}}-x_{2} \lambda_{x_{2}}\right] .
\end{aligned}
$$

Consider $X^{0} \in \mathfrak{S} \backslash \mathscr{T}$ and let $D$ be an optimal control of (OCP and $X(\cdot)$ the associated trajectory reaching the target set $\mathscr{T}$ in a time $t_{f} \geq 0$. Then, there exists an absolutelycontinuous map $\lambda:\left[0, t_{f}\right] \rightarrow \mathbb{R}^{5}$ and $\lambda^{0} \leq 0$ satisfying:

- the pair $\left(\lambda(\cdot), \lambda^{0}\right)$ is non-zero,

- the adjoint equation a.e. over $\left[0, t_{f}\right]$,

$$
\dot{\lambda}(t)=-\frac{\partial H}{\partial X}\left(X(t), \lambda(t), \lambda^{0}, D(t)\right),
$$

- the Hamiltonian condition a.e.:

$$
D(t) \in \operatorname{argmax}_{\omega \in\left[0, D_{\max }\right]} H\left(X(t), \lambda(t), \lambda^{0}, \omega\right),
$$

- the transversality condition at $t=t_{f}$ :

$$
\lambda\left(t_{f}\right) \in-N_{\mathscr{T}}\left(X\left(t_{f}\right)\right)
$$

where given a non-empty convex subset $K \subset \mathbb{R}^{5}, N_{K}(x)$ stands for the (convex) normal cone to $K$ at some point $x \in K$ (see, e.g., [21]). The adjoint equation (8) is equivalent to:

$$
\left\{\begin{aligned}
\dot{\lambda}_{s}= & \left(\rho_{1}^{\prime}(s) x_{1}+\rho_{2}^{\prime}(s) x_{2}\right) \lambda_{s}-\rho_{1}^{\prime}(s) \lambda_{q_{1}} \\
& -\rho_{2}^{\prime}(s) \lambda_{q_{2}}+D \lambda_{s} \\
\dot{\lambda}_{q_{1}}= & \mu_{1 \infty} \lambda_{q_{1}}-\mu_{1}^{\prime}\left(q_{1}\right) x_{1} \lambda_{x_{1}} \\
\dot{\lambda}_{x_{1}}= & \rho_{1}(s) \lambda_{s}-\mu_{1}\left(q_{1}\right) \lambda_{x_{1}}+D \lambda_{x_{1}} \\
\dot{\lambda}_{q_{2}}= & \mu_{2 \infty} \lambda_{q_{2}}-\mu_{2}^{\prime}\left(q_{2}\right) x_{2} \lambda_{x_{2}} \\
\dot{\lambda}_{x_{2}}= & \rho_{2}(s) \lambda_{s}-\mu_{2}\left(q_{2}\right) \lambda_{x_{2}}+D \lambda_{x_{2}} .
\end{aligned}\right.
$$

Recall that an extremal is a quadruplet $\left(X(\cdot), \lambda(\cdot), \lambda^{0}, D(\cdot)\right)$ such that $\left(\lambda(\cdot), \lambda^{0}\right) \neq 0$ and satisfying (1)-(8)-(9)-(10). In the case where $\lambda^{0}=0$, the extremal is said abnormal whereas if $\lambda^{0} \neq 0$, then the extremal is normal. Finally, since (OCP is autonomous, the Hamiltonian is conserved along any extremal, and because the terminal time is free, we obtain that $H=0$ along any extremal of the problem.

The transversality condition 10 can be explicited as follows. Recall that the target set is a half-space of $\mathbb{R}^{5}$ and because $X^{0} \in \mathfrak{S} \backslash \mathscr{T}$, we necessarily have $X\left(t_{f}\right) \in E$ where $E:=\left\{X \in \mathbb{R}^{5} ; x_{2}-\varepsilon x_{1}=0\right\}$ is the boundary of $\mathscr{T}$ (recall that $\left.X=\left(s, q_{1}, x_{1}, q_{2}, x_{2}\right)\right)$. At $X\left(t_{f}\right)$, the normal cone to $\mathscr{T}$ writes,

$$
N_{\mathscr{T}}\left(X\left(t_{f}\right)\right)=\mathbb{R}_{+}(0,0,-\varepsilon, 0,1)
$$

The inclusion 10 is then equivalent to,

$$
\lambda_{s}\left(t_{f}\right)=\lambda_{q_{1}}\left(t_{f}\right)=\lambda_{q_{2}}\left(t_{f}\right)=0,
$$

together with,

$$
\lambda_{x_{1}}\left(t_{f}\right)+\varepsilon \lambda_{x_{2}}\left(t_{f}\right)=0 .
$$

Actually, we can also prove that,

$$
\lambda_{x_{1}}\left(t_{f}\right)>0 \text { and } \lambda_{x_{2}}\left(t_{f}\right)<0 .
$$

As a consequence, the transversality condition 10 is equivalent to (12)-(13)-(14).

In order to exploit the Hamiltonian condition (9), it is useful to introduce the switching function which allows us to give the value of the control in terms of its sign. Doing so, let $\tilde{\Phi}$ be the switching function associated with the control $D(\cdot)$ :

$$
\tilde{\Phi}=\left(s_{i n}-s\right) \lambda_{s}-\left(x_{1} \lambda_{x_{1}}+x_{2} \lambda_{x_{2}}\right) .
$$

Since the Hamiltonian is linear w.r.t. the control, we get,

$$
\begin{aligned}
& \tilde{\Phi}(t)>0 \Rightarrow D(t)=D_{\max }, \\
& \tilde{\Phi}(t)<0 \Rightarrow D(t)=0,
\end{aligned}
$$

for a.e. $t \in\left[0, t_{f}\right]$. When the control $D(\cdot)$ is non-constant in every neighborhood of a time $t_{c} \in\left(0, t_{f}\right)$, we say that $t_{c}$ is a switching time, and one must have $\tilde{\Phi}\left(t_{c}\right)=0$. It may also happen that $\tilde{\Phi}$ vanishes over a sub-interval $\left[t_{1}, t_{2}\right]$ of $\left[0, t_{f}\right]$. In that case, we say that a singular arc occurs over $\left[t_{1}, t_{2}\right]$ and that the corresponding trajectory is singular.

Before investigating further singular arcs, let us give some properties of $\tilde{\Phi}$. 
Lemma 1: (i) The switching function $\tilde{\Phi}$ is continuously differentiable over $\left[0, t_{f}\right]$, and one has,

$$
\dot{\tilde{\Phi}}=\left(s_{\text {in }}-s\right)\left(\rho_{1}^{\prime}(s)\left[x_{1} \lambda_{s}-\lambda_{q_{1}}\right]+\rho_{2}^{\prime}(s)\left[x_{2} \lambda_{s}-\lambda_{q_{2}}\right]\right) .
$$

(ii) At the terminal time $t=t_{f}$, one has,

$$
\tilde{\Phi}\left(t_{f}\right)=\dot{\tilde{\Phi}}\left(t_{f}\right)=0
$$

We now study singular arcs which are essential in the optimal synthesis.

\section{Singular ARCS}

\section{A. Second derivative of the switching function}

We start by computing $\ddot{\tilde{\Phi}}$ (which exists almost everywhere). In particular, this will allow us to obtain the value of the singular control, i.e., the expression of $D$ along a singular arc (recall that along a singular arc, (9) is non-informative).

Let us define $\theta_{1}, \theta_{2}:[0, T] \rightarrow \mathbb{R}$ as,

$$
\begin{aligned}
& \theta_{1}:=\rho_{1}^{\prime}(s)\left[x_{1} \lambda_{s}-\lambda_{q_{1}}\right]+\rho_{2}^{\prime}(s)\left[x_{2} \lambda_{s}-\lambda_{q_{2}}\right], \\
& \theta_{2}:=\rho_{1}^{\prime \prime}(s)\left[x_{1} \lambda_{s}-\lambda_{q_{1}}\right]+\rho_{2}^{\prime \prime}(s)\left[x_{2} \lambda_{s}-\lambda_{q_{2}}\right] .
\end{aligned}
$$

To shorten, we did not write the dependency of $\theta_{1}, \theta_{2}, s, x_{1}, x_{2}, \lambda_{s}, \lambda_{q_{1}}$ and $\lambda_{q_{2}}$ w.r.t. $t$. As well, we shall hereafter omit the dependency w.r.t. some variables when there is no ambiguity. In view of (16) and (11), one can write,

$$
\dot{\tilde{\Phi}}=\left(s_{i n}-s\right) \theta_{1} \text { and } \dot{\lambda}_{s}=\theta_{1}+D \lambda_{s} \text {. }
$$

Next, observe that the derivative of $\theta_{1}$ satisfies,

$$
\begin{aligned}
\dot{\theta}_{1}= & \theta_{2} \dot{s}+\left[\rho_{1}^{\prime} x_{1}+\rho_{2}^{\prime} x_{2}\right] \theta_{1}+\lambda_{s}\left[x_{1} \mu_{1} \rho_{1}^{\prime}+x_{2} \mu_{2} \rho_{2}^{\prime}\right] \\
& -\rho_{1}^{\prime}\left[\mu_{1 \infty} \lambda_{q_{1}}-\mu_{1}^{\prime}\left(q_{1}\right) x_{1} \lambda_{x_{1}}\right] \\
& -\rho_{2}^{\prime}\left[\mu_{2 \infty} \lambda_{q_{2}}-\mu_{2}^{\prime}\left(q_{2}\right) x_{2} \lambda_{x_{2}}\right] .
\end{aligned}
$$

Therefore, we deduce that along any extremal, one has almost everywhere:

$$
\begin{aligned}
\ddot{\tilde{\Phi}}= & {\left[-\theta_{1}+\left(s_{i n}-s\right) \theta_{2}\right] \dot{s}+\left(s_{i n}-s\right)\left[\rho_{1}^{\prime} x_{1}+\rho_{2}^{\prime} x_{2}\right] \theta_{1} } \\
& +\left(s_{i n}-s\right) \lambda_{s}\left[x_{1} \mu_{1} \rho_{1}^{\prime}+x_{2} \mu_{2} \rho_{2}^{\prime}\right] \\
& -\left(s_{i n}-s\right) \rho_{1}^{\prime}\left[\mu_{1 \infty} \lambda_{q_{1}}-\mu_{1}^{\prime}\left(q_{1}\right) x_{1} \lambda_{x_{1}}\right] \\
& -\left(s_{i n}-s\right) \rho_{2}^{\prime}\left[\mu_{2 \infty} \lambda_{q_{2}}-\mu_{2}^{\prime}\left(q_{2}\right) x_{2} \lambda_{x_{2}}\right] .
\end{aligned}
$$

Next, recall that along a singular arc defined over a time interval $I=\left[t_{1}, t_{2}\right]$, the (so-called Legendre-Clebsch's) condition,

$$
\ddot{\tilde{\Phi}}_{\left.\right|_{D}} \geq 0,
$$

should be fulfilled over $I=\left[t_{1}, t_{2}\right]$. From the expression of $\ddot{\tilde{\Phi}}$ given by 19 , we obtain along a singular arc defined over a time interval $I=\left[t_{1}, t_{2}\right]$,

$$
\ddot{\tilde{\Phi}}_{\left.\right|_{D}}=\left(s_{\text {in }}-s\right)^{2} \theta_{2}=\left(x_{1} \lambda_{s}-\lambda_{q_{1}}\right) \frac{\left(\rho_{1}^{\prime \prime} \rho_{2}^{\prime}-\rho_{2}^{\prime \prime} \rho_{1}^{\prime}\right)}{\rho_{2}^{\prime}} .
$$

When Legendre-Clebsch's condition is fulfilled, we usually say that the singular arc is of turnpike type (see, e.g., [8]). In that case, the expression of the singular control follows from (19). First, let us set:

$$
\begin{aligned}
a(X, \lambda):= & \theta_{2}\left[\rho_{1} x_{1}+\rho_{2} x_{2}\right]-\lambda_{s}\left(x_{1} \mu_{1} \rho_{1}^{\prime}+x_{2} \mu_{2} \rho_{2}^{\prime}\right) \\
& -\rho_{1}^{\prime}\left[\mu_{1 \infty} \lambda_{q_{1}}-\mu_{1}^{\prime}\left(q_{1}\right) x_{1} \lambda_{x_{1}}\right] \\
& -\rho_{2}^{\prime}\left[\mu_{2 \infty} \lambda_{q_{2}}-\mu_{2}^{\prime}\left(q_{2}\right) x_{2} \lambda_{x_{2}}\right] .
\end{aligned}
$$

Now, let us state the following result:

Proposition 2: Suppose that an extremal is singular over $\left[t_{1}, t_{2}\right]$ and that Legendre-Clebsch's condition is fulfilled over $\left[t_{1}, t_{2}\right]$ with a strict inequality in 20 . Then, the singular control can be expressed as a feedback of state and adjoint variables as,

$$
D_{s}(X, \lambda):=\frac{a(X, \lambda)}{\left(s_{i n}-s\right) \theta_{2}} .
$$

Remark 1: Note that Legendre-Clebsch's condition (with a strict inequality) is equivalent to $\theta_{2}>0$ and that this condition is always verified over $\left[t_{c}, t_{f}\right]$ with $t_{c}$ close to $t_{f}$ (this follows from Assumption (1)).

\section{B. Discussion about the minimal time synthesis}

At $t=t_{f}$, the switching function and its derivative vanish (as a consequence of the transversality conditions (17)). In addition, we can observe (adapting slightly Proposition 2) that Legendre-Clebsch's condition (20) with a strict inequality is always satisfied in some small time interval $\left[t_{f}-\eta, t_{f}\right)$. Because of these elements, we suspect at first glance that a singular arc occurs on some time interval $\left[t_{c}, t_{f}\right]$ where $0 \leq t_{c}<t_{f}$ and that an optimal trajectory reaches $\mathscr{T}$ via a singular arc. Nevertheless, it is worth noticing that the conditions,

$$
\dot{\tilde{\Phi}}\left(t_{c}\right)=\tilde{\Phi}\left(t_{c}\right)=0
$$

are not equivalent to the occurrence of a singular arc starting at some time $t_{c}<t_{f}$ (contrary to what was suggested earlier in [12] and [6]). However, from extensive numerical simulations using direct optimization approaches (see Sect. $\mathrm{V}$ but also [12], [11]), we note that $\tilde{\Phi}$ and $\dot{\tilde{\Phi}}$ vanish in some significantly large time interval $\left[t_{c}, t_{f}\right]$ depending on the initial condition. To summarize, combining the theoretical analysis of $\mathrm{OCP}$ together with the numerical simulations of Sect. V] we believe that the minimal-time synthesis presents the following properties:

- For initial conditions in some subset $\mathfrak{S}^{\dagger} \subset \mathfrak{S}$, the structure of an optimal control is bang-singular (BS) or singular (S). Moreover, the singular path corresponding to $D_{s}$ exhibits a turnpike-type behavior in some neighborhood of $s=s_{c}$.

- For initial conditions outside $\mathfrak{S}^{\dagger}$, the structure of an optimal control may be of bang-type (B), of bangsingular-type (BS), or bang-bang-singular-type (BBS).

Remark 2: The BS or S behavior described previously seems the most common one. It is also the most compelling from a biological standpoint, since in practice, initial conditions start commonly sufficiently "far" from the target $\mathscr{T}$. This is typically the case observed when the initial culture is homogeneous, i.e., $x_{1}(0) / x_{2}(0) \approx 1$, requiring consequently a significant amount of time to achieve species separation. On the other hand, the other cases stand for more marginal situations, where for instance the optimization problem starts very close to the target $\mathscr{T}$. Typically, when $x_{1}(0)>>x_{2}(0)$ and $\mu_{1}\left(q_{1}(0)\right)>\mu_{2}\left(q_{2}(0)\right)$, it is observed that sometimes a bang arc (B) corresponding to $D(t)=0$ over $\left[0, t_{f}\right)$ steers the model trajectories to the target $\mathscr{T}$ in minimal-time. 
Remark 3: The turnpike behavior characterizing optimal trajectories $s(t), q_{1}(t), q_{2}(t)$, as well as their respective costates, when the optimal $t_{f}$ is sufficiently large (for suitable initial conditions, see Remark 2), is highlighted in [12].

\section{NUMERICAL OPTIMAL SYNTHESIS RESULTS}

We consider the biological parameters and functions given in Table I which verify the assumptions stated in Sect. II, as illustrated in Fig. 2 .

\begin{tabular}{|l|l|l|l|l|}
\hline$i$ & $\begin{array}{l}\mu_{i \infty} \\
(L / \text { day })\end{array}$ & $\begin{array}{l}k_{q_{i}} \\
\left(\mu \mathrm{mol} / \mu \mathrm{m}^{3}\right)\end{array}$ & $\begin{array}{l}\rho_{\text {im }} \\
\left(\mu \mathrm{mol} / \mu \mathrm{m}^{3} / \text { day }\right)\end{array}$ & $\begin{array}{l}K_{\text {si }} \\
(\mu \mathrm{mol} / \mathrm{L})\end{array}$ \\
\hline 1 & 1.7 & 1.75 & 9 & 0.3 \\
\hline 2 & 1.9 & 1.80 & 10 & 0.6 \\
\hline
\end{tabular}

DROOP PARAMETER VALUES.
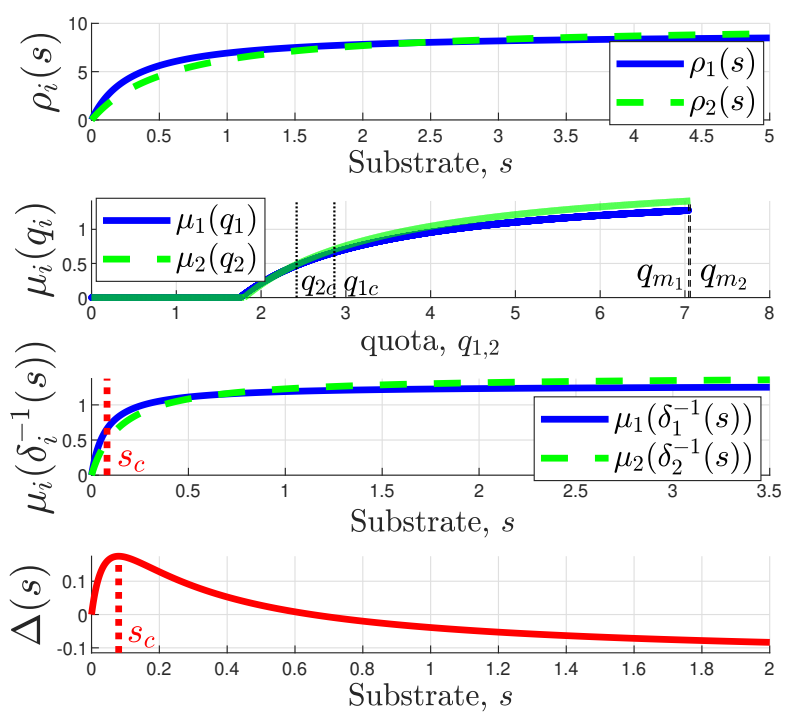

Fig. 2. Uptake and growth functions corresponding to the parameters in Table I] The value of $s$ maximizing $\Delta(s)$ is $s_{c}=0.08$.

We consider $s_{\text {in }}=5 \mu \mathrm{mol} / L$ and define a contamination threshold $\varepsilon=0.05$ in the target $\mathscr{T}$.

A direct optimization method is performed using the software Bocon $^{2}$ ([16]). In Bocop all the state and the control variables are discretized. The dilution-rate $D$ is discretized over $\left[0, D_{\max }\right]$ such that it is piecewise constant on the timeinterval subdivision. The state dynamics are also discretized, through a scheme of ordinary differential equations (ODE) decomposition (e.g., Euler method, Runge-Kutta, etc.). The numerical direct method transforms (OCP) into a nonlinear programming problem (NLP) in finite dimension. Here we use in Bocop a predefined variant of Lobatto methods for numerical integration of ODEs ([17], [18]). It is an interior point approach, with a discontinuous collocation method of Lobatto's type of sixth order time-discretization (Labatto III-C formula ([19])). The tolerance for NLP solver in Bocop is tuned to $10^{-20}$ and the time-discretization is fixed to 150 steps. The upper-bound on the control is $D_{\max }=1.5$, which is designed right above the maximum effective growth rate ([12]) of microalgae (see, $\mu_{i}\left(\delta_{i}^{-1}(s)\right), i=1,2$, Fig. 2).

In this example, we illustrate the common behavior described in the previous section, i.e., starting from an homogeneous culture where $x_{1}(0) / x_{2}(0)=1$. More precisely, we consider in this example the initial conditions given by, $s^{0}=2 \mu \mathrm{mol} / L, q_{1}^{0}=2 \mu \mathrm{mol} / \mu \mathrm{m}^{3}, x_{1}^{0}=0.1 \mu \mathrm{m}^{3} / L$, $q_{2}^{0}=2 \mu \mathrm{mol} / \mu \mathrm{m}^{3}, x_{2}^{0}=0.1 \mu \mathrm{m}^{3} / L$. Using Bocop, we get the minimal-time $t_{f}$ required to reach the target $\mathscr{T}$ : $t_{f}=18.8846$ days, $x_{1}\left(t_{f}\right)=1.78716 \mu \mathrm{m}^{3} / L$, and, $x_{2}\left(t_{f}\right)=$ $0.08935 \mu \mathrm{m}^{3} / \mathrm{L}$. The corresponding optimal control $D$ is given in Fig. 3, which is bang(0)-singular (BS). The optimal trajectories given by Bocop are given in Fig. 4, where we clearly observe a turnpike-like behavior in the trajectories $s$, $q_{1}$ and $q_{2}$, as well as in their respective co-states in Fig. 5 (see also [12] for further examples). We note that $\lambda_{s}$ (Fig. 5 is zero on the singular arc, over $\left[t_{s}, t_{f}\right]$, where $t_{s}$ is the switching instant of the control.

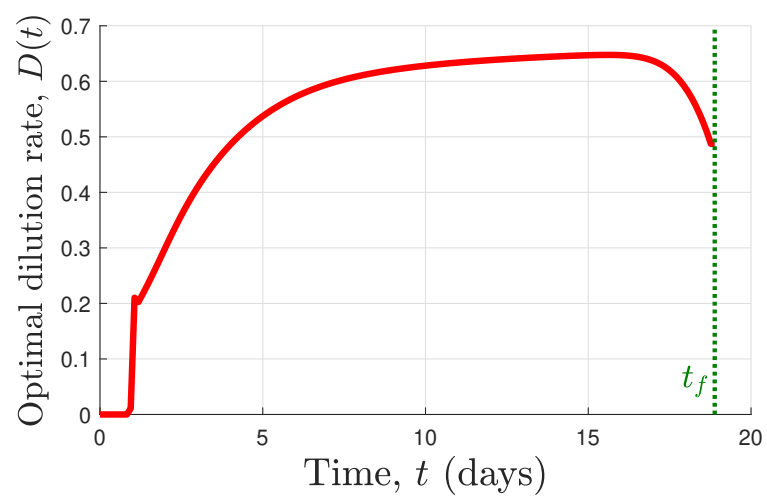

Fig. 3. The optimal control bang $(0)$-singular over $\left[0, t_{f}\right]$

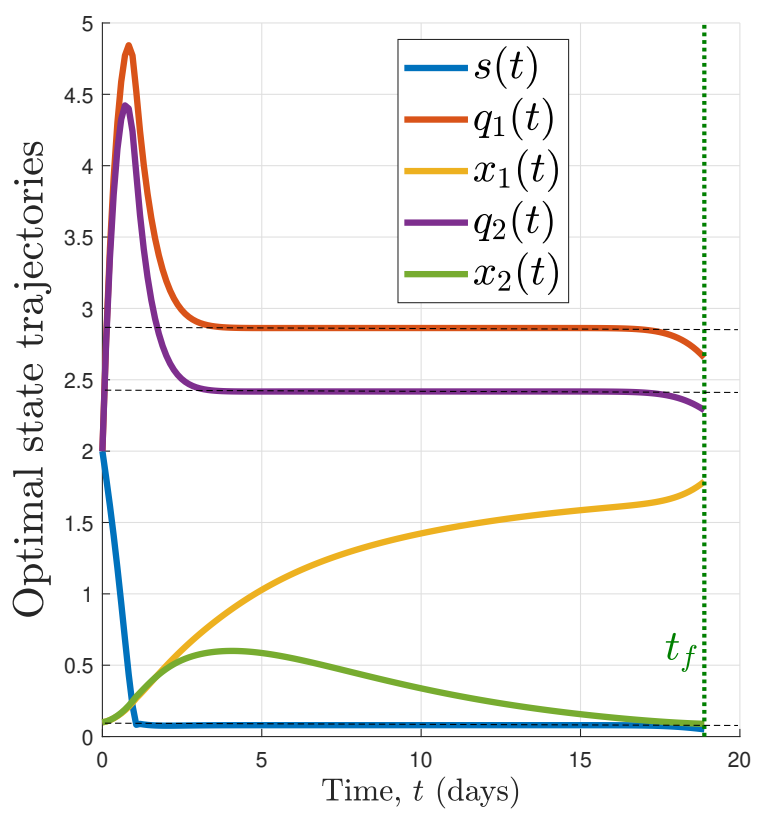

Fig. 4. Optimal trajectories starting from $s^{0}=2 \mu \mathrm{mol} / L, q_{i}^{0}=2$ $\mu \mathrm{mol} / \mu \mathrm{m}^{3}, x_{i}^{0}=0.1 \mu \mathrm{m}^{3} / L, i=1,2$, steered by the control in Fig. 3 


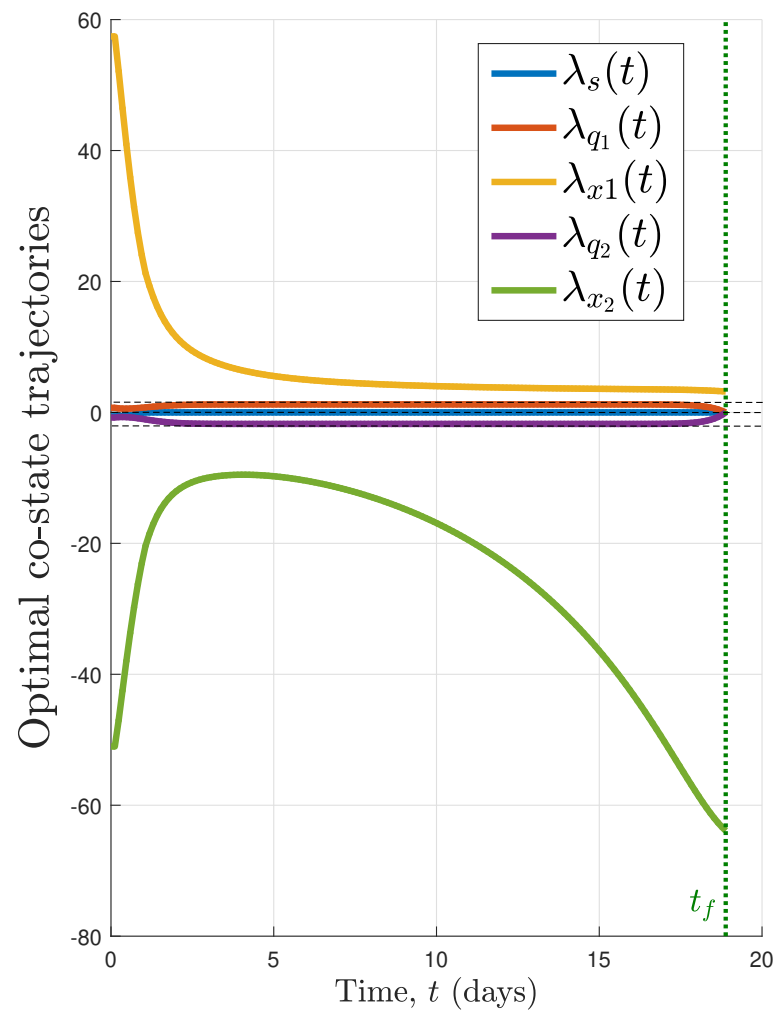

Fig. 5. The optimal co-state trajectories obtained using Bocop.

\section{CONCLUSION}

The minimal-time OCP for two species in competition described by the five-dimensional Droop's model turns out to be significantly more complicated than for the Monod model with two species (see [6]) and its extension with $n \geq 3$ species [7]. We revisited Problem (OCP) tackled in [12] in order to provide new insights into the optimal synthesis, in particular by checking Legendre-Clebsch's condition. Finding an optimal feedback control in this high dimensional framework appears to be a challenging issue. Future work will focus on the determination of closed loop sub-optimal control strategies which can be experimentally implemented. As well, the occurrence of a singular arc in a left neighborhood of the terminal time will deserve more attention.

\section{ACKNOWLEDGEMENT}

This research benefited from the support of the FMJH Program PGMO and from the support to this program from EDF-THALESORANGE.

\section{REFERENCES}

[1] Socransky, S.S. and Haffajee, A.D., 2005. Periodontal microbial ecology., Periodontology 2000, 38(1), pp.135-187.

[2] Wijffels, R.H., Barbosa, M.J. and Eppink, M.H., 2010. Microalgae for the production of bulk chemicals and biofuels., Biofuels, Bioproducts and Biorefining: Innovation for a sustainable economy, 4(3), pp.287295.

[3] Mata, T.M., Martins, A.A. and Caetano, N.S., 2010. Microalgae for biodiesel production and other applications: a review., Renewable and sustainable energy reviews, 14(1), pp.217-232.
[4] Morales, M., Collet, P., Lardon, L., Hélias, A., Steyer, J.P. and Bernard, O., 2019. Life-cycle assessment of microalgal-based biofuel., Biofuels from Algae, pp.507-550.

[5] Bonnefond, H., Grimaud, G., Rumin, J., Bougaran, G., Talec, A., Gachelin, M., Boutoute, M., Pruvost, E., Bernard, O. and Sciandra, A., 2017. Continuous selection pressure to improve temperature acclimation of Tisochrysis lutea., PloS one, 12(9).

[6] Bayen, T., \& Mairet, F., 2014. Optimization of the separation of two species in a chemostat., Automatica, 50(4), pp.1243-1248.

[7] Bayen, T., \& Mairet, F., 2017. Optimization of strain selection in evolutionary continuous culture, International Journal of Control, 90(12), pp.2748-2759.

[8] Boscain, U. \& Piccoli, B., 2004. Optimal Syntheses for Control Systems on 2-D Manifolds, Springer SMAI, vol. 43.

[9] Droop, M.R., 1968. Vitamin $B_{12}$ and marine ecology. IV. The kinetics of uptake growth and inhibition in Monochrysis lutheri. J. Mar. Biol. Assoc. 48 (3), pp.689-733.

[10] Droop, M.R., 1973. Some thoughts on nutrient limitation in algae. Journal of Phycology, 9(3), pp.264-272.

[11] Djema, W., Giraldi, L., Bernard, O., 2019. An Optimal Control Strategy Separating Two Species of Microalgae in Photobioreactors., Conference on Dynamics and Control of Process Systems, including Biosystems-12th DYCOPS., IFAC-PapersOnLine, Vol.52, Issue 1, pp.910-915. https://doi.org/10.1016/j.ifacol.2019.06.178.

[12] Djema, W., Bernard, O., Giraldi, L., 2020. Separating Two Species of Microalgae in Photobioreactors in Minimal Time., Journal of Process Control, Vol.87, pp.120-129. https://doi.org/10.1016/j.jprocont.2020.01.003.

[13] Pontryagin, L.S., Boltyanskiy, V.G., Gamkrelidze, R.V., Mishchenko, E.F., 1964. Mathematical theory of optimal processes., New York, NY Springer.

[14] Caperon, J., \& Meyer, J., 1972. Nitrogen-limited growth of marine phytoplankton - II. Uptake kinetics and their role in nutrient limited growth of phytoplankton. In Deep Sea Research and Oceanographic Abstracts, Vol.19, No. 9, pp. 619-632.

[15] Trélat, E. and Zuazua, E., 2015. The turnpike property in finitedimensional nonlinear optimal control., Journal of Differential Equations, 258(1), pp.81-114.

[16] Bonnans, F. J., Giorgi, D., Grelard, V., Heymann, B., Maindrault, S. Martinon, P., Tissot, O., Liu, J., 2017. BOCOP: an open source toolbox for optimal control - A collection of examples., Team Commands, Inria Saclay, Technical Reports, http://bocop.org.

[17] Betts, J. T., 2010. Practical methods for optimal control and estimation using nonlinear programming., Siam, Advances in Design \& Control, 2nd Edition, Vol.19, p.427.

[18] Biegler, L. T., 2010. Nonlinear Programming: Concepts, Algorithms, and Applications to Chemical Processes., MPS-SIAM Series on Optimization (Book 10), SIAM-Society for Industrial and Applied Mathematics, p.415.

[19] Hairer, E., Norsett, S.P., Wanner, G., Solving ordinary differential equations., 1, Nonstiff problems. Springer-Vlg.

[20] Smith, H.L. and Waltman, P., The theory of the chemostat, Dynamics of microbial competition, Cambridge University Press, 1995.

[21] Vinter, R., Optimal Control, Systems and Control: Foundations and Applications, Birkhauser, 2000. 